\title{
Metabolic Considerations in Atrial Fibrillation - Mechanistic Insights and Therapeutic Opportunities -
}

\author{
Masahide Harada, MD, PhD; Jonathan Melka, PhD; \\ Yoshihiro Sobue, MD, PhD; Stanley Nattel, MD
}

\begin{abstract}
Atrial fibrillation (AF) is the most common sustained arrhythmia in clinical practice and is associated with morbidity and mortality. Over the past 2 decades, there have been major advances in understanding AF pathophysiology, but important knowledge gaps, particularly about targetable basic mechanisms, remain. Recent metabolomic and proteomic studies have shown changes in the expression of molecules involved in metabolic pathways in human and experimental AF, indicating a role for metabolic alterations in $\mathrm{AF}$ pathophysiology. AF is characterized by irregular high-frequency excitation and contraction that affect atrial energy demands, circulation and oxygen supply, and change the balance between metabolic demand and supply, causing metabolic stress. Here, we review the information available about AF-induced metabolic changes and their pathophysiological contribution. We also discuss the possibilities of developing novel therapeutic strategies that act by modulating cardiac metabolic processes during AF.
\end{abstract}

Key Words: Atrial fibrillation; Metabolism; Novel pharmacotherapy

A trial fibrillation (AF) is the most frequent arrhythmia in clinical practice and is associated with increased morbidity and mortality. ${ }^{1}$ However, effective therapeutic approaches are limited and novel mechanistic understanding is required for therapeutic innovation. ${ }^{2}$ The maintenance of cardiac work requires oxygen and nutrient supply that matches the needs created by energy expenditure (Figure 1); accordingly, excitation-contraction coupling and ion channel/pump integrity are closely linked to cellular metabolic conditions. ${ }^{3} \mathrm{AF}$-induced irregular high-frequency excitation and contraction alter atrial hemodynamics, oxygen delivery, and energy supply, all of which change the metabolic state. Recent work has demonstrated substantial changes in metabolism-related molecules in AF.4-7 Here, we review the evidence regarding the pathophysiological role of AF-associated cellular metabolic changes and their potential value as therapeutic targets.

\section{Cardiomyocyte Energy Metabolism}

Adenosine triphosphate (ATP) is an essential energy source for cardiac work, including excitation, contraction, relaxation, and molecular synthesis/degradation. ATP is produced as a principal product of the metabolism of several substrates, including fatty acids (FAs), glucose, amino acids, and ketone bodies, but FAs and glucose are the principal energy substrates in cardiomyocytes. The energy production pathways include (1) glycolysis (in the cytosol), and (2) glucose oxidation, (3) FA oxidation, (4) the tricarboxylic acid (TCA) cycle, and (5) electron-transport chain (ETC) metabolism in the mitochondria (Figure 2). During energy production, nicotinamide adenine dinucleotide (NADH) and flavin adenine dinucleotide $\left(\mathrm{FADH}_{2}\right)$ are generated and directed into the mitochondrial ETC to produce ATP via redox reactions. The mitochondrial redox reaction also produces reactive oxygen species (ROS) as a byproduct (Figure 2). ${ }^{8-11}$

ATP is hydrolyzed (catalyzed) by ATPase into adenosine diphosphate $(\mathrm{ADP})+1$ inorganic phosphate $(\mathrm{Pi})$ or adenosine monophosphate (AMP)+ $2 \mathrm{Pi}$, liberating energy. Specific energy-producing ATPases include actomyosinATPase in myofilaments, sarcoplasmic reticulum (SR) $\mathrm{Ca}^{2+}$-ATPase-2a (SERCA2a), and sarcolemmal $\mathrm{Na}^{+} / \mathrm{K}^{+}$ATPase.

Cardiomyocyte contraction is determined by energyrequiring contractile and $\mathrm{Ca}^{2+}$ dynamics. $\mathrm{Ca}^{2+}$-sensitive actomyosin-ATPase directly provides the energy for myofilament movement. An additional energy-storage compound, phosphocreatine (PCr), generates ATP for actomyosin-ATPase during rapidly increased cardiac work

Received September 27, 2017; accepted September 29, 2017; released online October 24, 2017

Department of Cardiology, Fujita Health University, Toyoake (M.H.), Japan; Department of Medicine and Research Center, Montreal Heart Institute, Montreal, Quebec (J.M., Y.S., S.N.); Université de Montréal, Montreal, Quebec (J.M., Y.S., S.N.); Department of Pharmacology and Therapeutics, McGill University, Montreal, Quebec (J.M., Y.S., S.N.), Canada; and Institute of Pharmacology, West German Heart and Vascular Center, Faculty of Medicine, University Duisburg-Essen, Essen (J.M., Y.S., S.N.), Germany

Present address: Department of Cardiology, Gifu Heart Center, Gifu (Y.S.), Japan.

The first two authors contributed equally to this work (M.H., J.M.).

Mailing address: Stanley Nattel, MD, Research Center, Montreal Heart Institute, 5000 Belanger Street East, Montreal, Quebec H1T 1C8, Canada. E-mail: stanley.nattel@icm-mhi.org

ISSN-1346-9843 All rights are reserved to the Japanese Circulation Society. For permissions, please e-mail: cj@j-circ.or.jp 


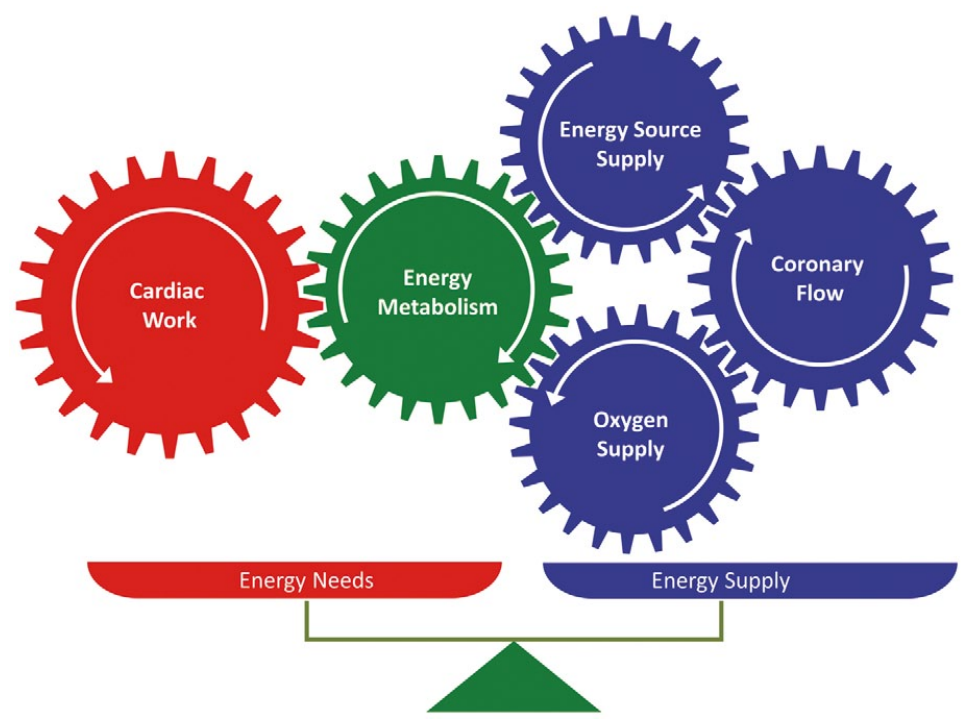

Figure 1. Metabolic gears and cardiac work. Coronary blood flow delivers energy sources and oxygen, which allow for the energy metabolism that drives cardiac work production.



Figure 2. Energy production system and utilization in cardiomyocytes. CK, creatine kinase; CPT-1, carnitine palmitoyl transferase-1; Cr, creatinine; ETC, electron transport chain; FA, fatty acid; FAT, FA transporter; FADH2, flavin adenine dinucleotide; GLUT 1/4, glucose transporter type-1/4; NADH, nicotinamide adenine dinucleotide; PCr, phosphocreatine; PDH, pyruvate dehydrogenase; ROS, reactive oxygen species; TCA cycle, tricarboxylic acid cycle.

situations under the control of creatine kinase (CK). SERCA2a drives $\mathrm{Ca}^{2+}$ from the cytosol into the SR to allow muscle relaxation and maintain SR $\mathrm{Ca}^{2+}$-stores. The $\mathrm{Na}^{+}$/ $\mathrm{K}^{+}$-ATPase pumps $3 \mathrm{Na}^{+}$out of the cell while transporting $2 \mathrm{~K}^{+}$into the cell, maintaining the essential transmembrane ionic gradients in the face of ion movements that occur during the action potential (Figure 2)..$^{8-11}$

\section{Glycolysis and Glucose Oxidation}

Glucose is taken into the cell via the sarcolemmal glucosetransporters types 1 and 4 (GLUT1/4) and metabolized via glycolysis in the cytosol. Phosphofructokinase catalyzes 


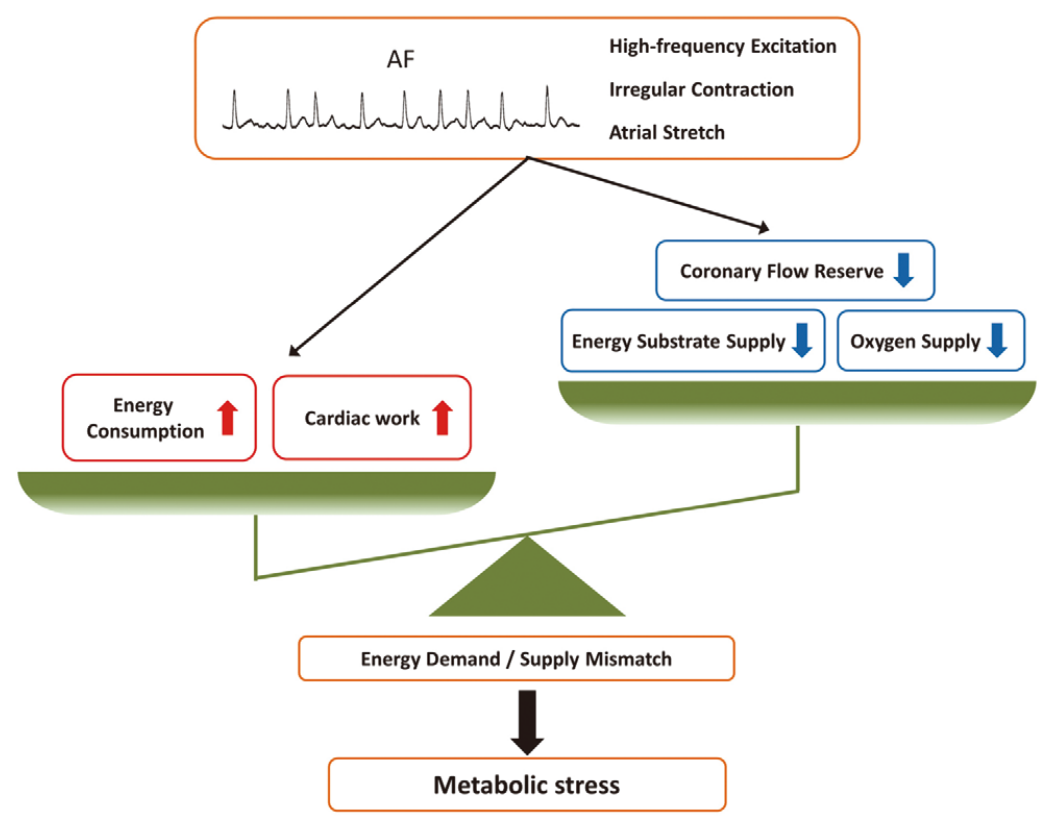

Figure 3. Metabolic imbalance and metabolic stress in atrial fibrillation (AF).

the phosphorylation of fructose-6-phosphate to fructose1,6-bisphosphate, a key regulatory step in glycolysis. Glycolysis produces 2 ATPs and 1 NADH independently of oxygen. Although on its own glycolysis generates limited ATP, glycolytic ATP generation becomes critical under anaerobic conditions. If oxidative metabolism fails under anaerobic conditions, pyruvate cannot be oxidized but builds up and and is converted to lactic acid, which causes cellular acidosis and ischemic consequences. Pyruvate, the endproduct of glycolysis, moves into the mitochondria and is catalyzed to produce acetyl-CoA and NADH by pyruvate dehydrogenase $(\mathrm{PDH})$, a rate-limiting enzyme for glucose oxidation. PDH activity depends on its phosphorylation level; it is inhibited by PDH-kinase and activated by PDH-phosphatase (Figure 2). ${ }^{\mathbf{8}-11}$

\section{FA Metabolism}

Circulating FAs enter cardiomyocytes via the FA transporter, FAT/CD36. Carnitine palmitoyl transferase-1 (CPT-1) allows FA entry into mitochondria for FA oxidation. Each FA oxidation cycle produces acetyl-CoA, $\mathrm{NADH}$ and $\mathrm{FADH}_{2}$. Malonyl-CoA is a potent CPT-1 suppressor and is inhibited by acetyl-CoA carboxylase (ACC). Both FA oxidation and glucose oxidation produce acetyl-CoA, and are interdependent such that increased FA oxidation suppresses glucose oxidation and vice versa (Figure 2)..$^{8-11}$

\section{TCA Cycle}

Acetyl-CoA is the main substrate for the TCA cycle, a series of metabolic processes that generate energy sources (guanosine-triphosphate, GTP, NADH, and $\mathrm{FADH}_{2}$ ) via oxidative reactions. NADH and $\mathrm{FADH}_{2}$ feed into the mitochondrial ETC to generate ATP (Figure 2). ${ }^{\mathbf{8 - 1 1}}$

\section{Mitochondrial ETC}

The ETC is the final metabolic process in the inner mitochondrial membrane. The ETC requires the coordination of 4 enzyme complexes (designated I-IV) and an ATP synthase (F0F1-ATPase). Complexes I-IV transfer electrons from electron donors to acceptors via redox reactions. The final electron acceptor is oxygen, producing $\mathrm{CO}_{2}$. The ETC extrudes protons $\left(\mathrm{H}^{+}\right)$from the mitochondrial matrix to the intermembrane space to create a $\mathrm{H}^{+}$-gradient across the mitochondrial inner membrane, driving F0F1-ATPase and synthesizing ATP (Figure 2).$^{\mathbf{8}-11}$

\section{Cardiac Work Efficiency}

Cardiac work efficiency is defined as the ratio of external work to oxygen consumption. FA metabolism generates more ATP but uses more oxygen than glucose metabolism. For example, the complete oxidation of $1 \mathrm{FA}$ palmitate molecule generates 105 ATP molecules and consumes 46 atoms of oxygen $(105 / 46=2.28)$, whereas oxidation of 1 glucose molecule generates 31 ATPs and consumes 12 atoms of oxygen $(31 / 12=2.58)$. FA oxidation generates NADH and $\mathrm{FADH}_{2}$ but glucose metabolism only generates NADH. In the mitochondrial ETC, NADH oxidation at Complex $\mathrm{I}$ is coupled to ATP production, whereas $\mathrm{FADH}_{2}$ oxidation bypasses Complex I and pumps fewer protons across the inner mitochondrial membrane. ${ }^{8}$

\section{Hemodynamics and Energy Demands in AF}

A variety of studies suggest a role for relative ischemia in AF. Depletion of high-energy phosphates (ATP and/or $\mathrm{PCr}$ ) and reduced activity of phosphotransfer enzymes occur in pacing-induced heart failure (HF) dogs with an AF substrate. ${ }^{11}$ Myofilament loss, glycogen accumulation, altered mitochondrial morphology, SR fragmentation, and nuclear chromatin dispersion resembling hibernating myocardium occur in goats with AF initially induced by electrical stimulation but subsequently maintained by electrical remodeling. ${ }^{12}$ Pigs subjected to rapid atrial pacing to mimic AF show increased atrial myocardial perfusion but also enhanced oxygen extraction. ${ }^{13}$ Increased venous 
lactate concentrations in the atrium, indicative of relative ischemia, result. ${ }^{13}$ ATP concentrations tend to decrease during acute stretch-related AF in rabbits. ${ }^{\mathbf{1 4}}$

Abnormalities in atrial blood supply have been shown by several studies. AF acutely increases atrial oxygen consumption while causing atrial distention, higher atrial pressures and lower atrial flow reserve. ${ }^{15}$ A study using intracoronary Doppler flow measurements showed impaired atrial perfusion and limited coronary flow reserve in lone AF patients, suggesting microvascular dysfunction. ${ }^{\mathbf{1 6}}$

Pacing-induced AF acutely increases mitochondrial F0F1-ATPase activity in sheep atria. ${ }^{17}$ Acute AF in goats decreases PCr without changes in ATP and metabolic enzyme activity, suggesting increased myofibril energy consumption. ${ }^{18}$ Thus, the literature indicates that AF is associated with metabolic changes related to decreased oxygen/nutrient delivery and/or increased energy demand.

Figure 3 summarizes the various factors that have been implicated in AF-related metabolic stress. These include changes increasing metabolic needs (enhanced cardiac work and energy consumption) as well as alterations that limit energy supply (limitations on coronary reserve, energy substrate supply and oxygen delivery).

\section{Cellular Metabolic Changes in AF}

\section{Fetal Phenotype}

During fetal development, glycolysis is a major energy source for proliferating cardiomyocytes. FA oxidation becomes predominant as cardiomyocytes mature and mitochondrial oxidative capacity increases. Glucose metabolism is more energy efficient, so with pathological stresses, energy metabolism switches to a more fetal phenotype. ${ }^{19}$

A transcriptomic study demonstrated that patients with permanent AF have more fetal phenotypes of metabolismrelated gene expression than patients in sinus rhythm, showing a marked increase in glycolysis-related gene expression. ${ }^{4}$ The activity of 1,6-bisphosphate aldolase, a glycolytic enzyme, increases in permanent AF patients (in whom AF continues indefinitely). ${ }^{20}$ Decreased glycolytic endproducts (alanine, lactate) relative to FA metabolism endproducts (acetate) correlate positively with the early onset of postoperative AF (POAF) after cardiac surgery, suggesting that decreased glucose metabolism may facilitate AF. ${ }^{\mathbf{2 0}}$

Myofilament isoform-switching occurs from $\alpha$-myosin heavy chain $(\alpha-\mathrm{MHC})$ to $\beta$-MHC in atrial tissues from AF patients. ${ }^{21} \alpha$-MHC (predominant in adult cardiomyocytes) produces higher-velocity muscle movement at a cost of more ATP and oxygen consumption than $\beta$-MHC (which predominates in embryonic hearts), so this switch improves metabolic economy. In addition, $\beta$-chain tropomyosin (slow-contracting myofilament with low energy consumption) and myosin light-chain embryonic muscle/atrial isoform expression increase in the atrial tissue of permanent AF patients. ${ }^{20}$

\section{Increased Ketone Body Metabolism}

Ketone bodies (acetoacetate, $\beta$-hydroxybutyrate, and acetone) are energy substrates produced by the liver from FAs and amino acids, and utilized by extrahepatic organs for energy production. Persistent (continuing $>1$ week) AF patients show increased $\beta$-hydroxybutyrate generation, with an increase in ketogenic amino acids (tyrosine and glysine) and 3-oxoacid-CoA transferase (a key enzyme for ketolytic energy production), suggesting increased ketone- body metabolism in AF, likely as an anaplerotic mechanism. ${ }^{5}$

\section{AMPK Activation}

AMP-activated protein kinase (AMPK) acts as an energy sensor and regulates cellular metabolism. AMPK is activated (phosphorylated) by upstream kinases (e.g., liver kinase B1 (LKB1), calmodulin kinase kinase) in response to energy depletion. AMPK compensates for energy depletion by increasing energy production and suppressing energy consumption.22 AMPK promotes FA oxidation by inhibiting ACC, the rate-limiting enzyme for malonyl-CoA. AMPK activation increases the expression of GLUT1/4 and promotes its trafficking into the sarcolemma, increasing glucose uptake. AMPK also phosphorylates phosphofructokinase-1, enhancing glycolysis. ${ }^{22}$ AMPK is activated in atrial tissues of dogs with electrically maintained AF and in canine atrial cardiomyocytes subjected to increased rate pacing. ${ }^{23}$ AMPK activation has also been shown in the atrial myocardium of AF patients compared with sinusrhythm controls, associated with increased FAT/CD36 expression and FA uptake. ${ }^{24}$

\section{Mitochondrial Dysfunction and ROS Generation}

AF has been reported to impair mitochondrial function, decreasing ATP production. AF-related mitochondrial dysfunction causes redox imbalance, increasing ROS production. ${ }^{25}$ Excess ROS injures vital genes and proteins, impairs cardiomyocyte function, and promotes AF-related remodeling associated with inflammation. ${ }^{26,27}$ Mitochondrial ultrastructural changes occur in the atrial tissue of AF goats. ${ }^{28}$ Rapid atrial pacing increases the expression of 3-nitrotyrosine, an oxidant agent, in canine atrial tissues. ${ }^{29}$ Mitochondrial morphology changes (i.e., pale, swollen) in cardiomyocyte cell-lines that are subjected to rapid pacing, accompanied by decreased ATP production and increased ROS production; high-frequency excitation per se causes mitochondrial dysfunction. ${ }^{30}$

Altered transcription of mitochondrial oxidative phosphorylation-related proteins and increased myofilament oxidation have been shown in permanent AF patients. ${ }^{21,31}$ Downregulation of ETC activity, increased proton leakage, and increased ROS production are also identified in AF patients undergoing cardiac surgery. ${ }^{32,33}$ Mitochondrial Complex II/III activity is decreased in permeabilized atrial fibers obtained from patients who developed POAF, corresponding to decreased expression of the gene cluster for mitochondrial oxidative phosphorylation. ${ }^{34} \mathrm{Cox} 5 \mathrm{~b}$, an enzyme complex of mitochondrial ETC, is responsible for the biosynthesis of ATP. Cox $5 b$ protein expression decreases in atrial tissue from AF patients compared with sinus-rhythm controls, suggesting impaired mitochondrial ETC function and energy production. ${ }^{7}$

Mitochondria possess their own mitochondrial DNA (mtDNA), encoding proteins required for essential functions. Patients with mtDNA deletions have increased AF prevalence and decreased concentrations of atrial ATP. ${ }^{35}$ On the other hand, AF patients have increased mtDNA mutation rates and evidence of atrial oxidative injury. ${ }^{36}$ In patients undergoing cardiac surgery ( 22 children/adolescents and 66 adults), mtDNA deletions were increased in the adult AF patients compared with those in sinus rhythm. Pediatric and adolescent patients did not show mtDNA deletions; thus, mtDNA deletion seems to be associated with aging. ${ }^{37}$ 


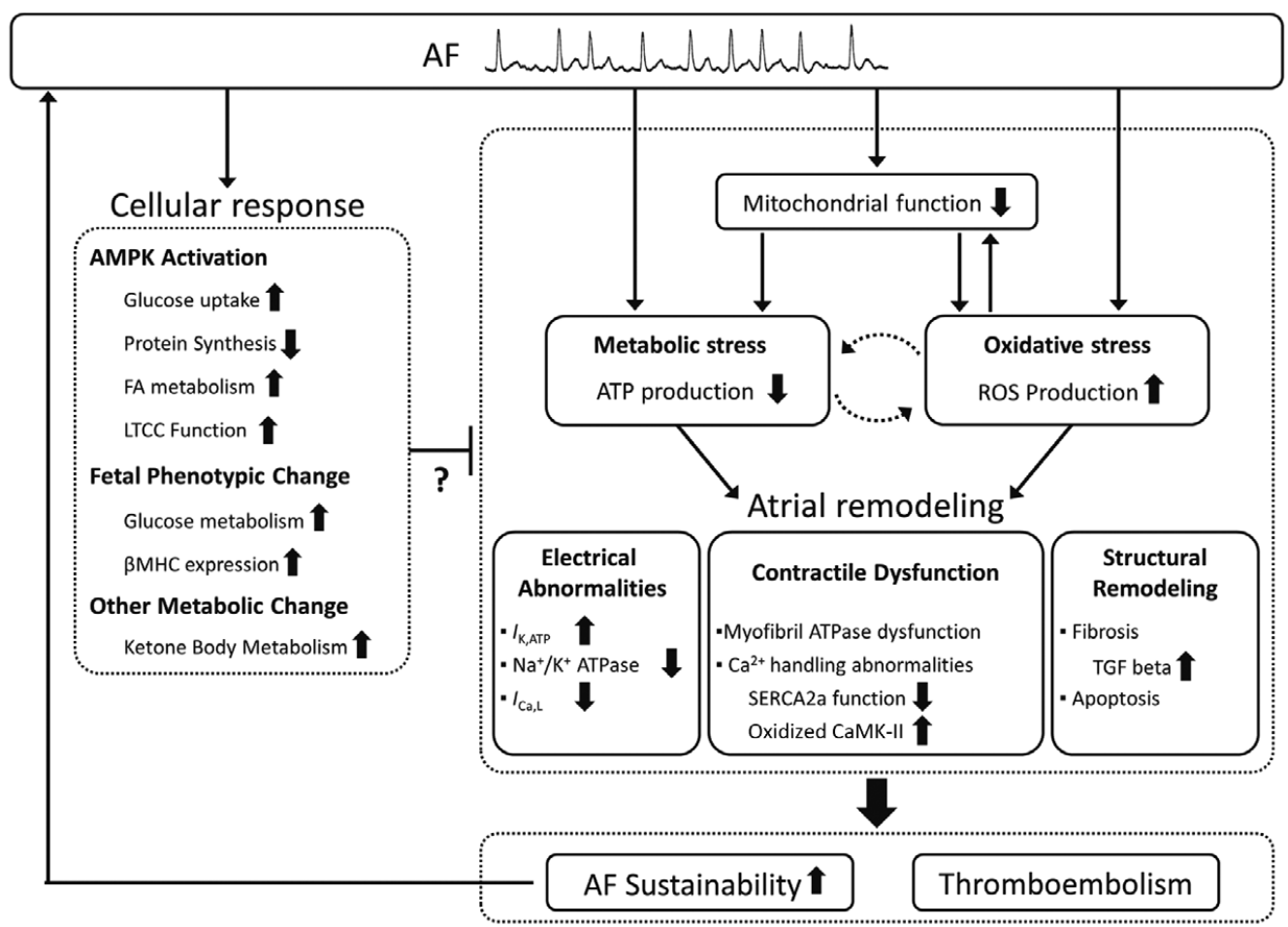

Figure 4. Contribution of energetic dysfunction to pathophysiology of atrial fibrillation (AF). AMPK, adenosine monophosphateactivated protein kinase; $\beta$-MHC, $\beta$-myosin heavy chain; FA, fatty acid; TGF- $\beta$, transforming growth factor- $\beta$; ROS, reactive oxygen species; SERCA2a, sarcoplasmic reticulum $\mathrm{Ca}^{2+}$-ATPase type $2 \mathrm{a}$.

\section{Atrial Remodeling Associated With Metabolic Stress}

\section{Electrophysiological Changes}

The sarcolemmal ATP-sensitive $\mathrm{K}^{+}$channel (IK,ATP) opens when the intracellular ATP concentration decreases. Increased $I_{\mathrm{K}, \mathrm{ATP}}$ hyperpolarizes the membrane and shortens the action potential duration (APD), facilitating AFpromoting reentry. IK,ATP is also activated by 1,3-bisphosphoglycerate, a glycolytic intermediate produced by the catalysis of glyceraldehyde 3-phosphate dehydrogenase (GAPDH). ${ }^{38}$ Glibenclamide, a IK,ATP inhibitor, does not affect AF-induced electrical remodeling, arguing against a role for $I_{\mathrm{K}, \mathrm{ATP}}{ }^{39} \mathrm{IK}_{\mathrm{K}, \mathrm{ATP}}$ channel subunits (Kir 6.2) and $I_{\mathrm{K}, \mathrm{ATP}}$ current density were decreased in persistent AF patients, possibly to counteract AF-induced APD shortening. ${ }^{\mathbf{4 0 , 4 1}}$

In a computational model of $\mathrm{AF}$, altered $\mathrm{Na}^{+} / \mathrm{K}^{+}$-ATPase function changed the APD90, APD restitution, and dominant frequency of spiral-wave reentry. ${ }^{42}$ However, Workman et al reported no difference in the $\mathrm{Na}^{+} / \mathrm{K}^{+}$-ATPase current in atrial cardiomyocytes from patients with vs. those without persistent AF. ${ }^{\mathbf{4 3}}$ Tran et al reported that increased atrial $\mathrm{Na}^{+} / \mathrm{K}^{+}$-ATPase expression and plasma $\mathrm{K}^{+}$-concentration increase the risk of POAF. ${ }^{44}$

Atrial cardiomyocyte metabolic stress reduces the L-type $\mathrm{Ca}^{2+}$ current, SR Ca ${ }^{2+}$ stores and cellular contractility. ${ }^{23}$ AMPK activation antagonizes these effects, and may be important for limiting arrhythmogenic APD shortening in AF patients. ${ }^{23}$ Impaired mitochondrial energy production induces $\mathrm{Ca}^{2+}$ transient (CaT) alternans; SERCA2a upregu- lation attenuates this phenomenon. ${ }^{45} \mathrm{Ca}^{2+} /$ calmodulin kinase type-II (CaMK-II) is an upstream regulator of $\mathrm{Ca}^{2+}$ handling proteins, and augments cellular $\mathrm{Ca}^{2+}$ dynamics. Mitochondrial ROS directly oxidize the enzyme regulatory domain of CaMK-II and induce CaMK-II activation. ${ }^{46}$ Sustained CaMK-II activation leads to abnormal $\mathrm{Ca}^{2+}$ homeostasis, SR $\mathrm{Ca}^{2+}$ leakage and the induction of atrial triggered activity that is implicated in AF initiation (Figure 4). ${ }^{2}$

\section{Contractile Remodeling}

AF increases the risk of stroke and thromboembolism in association with cellular hypocontractility.$^{47}$ Cardiomyocyte contraction depends on cellular $\mathrm{Ca}^{2+}$ dynamics, regulated by multiple $\mathrm{Ca}^{2+}$-handling proteins. Actomyosin-ATPase and SERCA2a function are linked to cellular metabolism. The $\mathrm{Ca}^{2+}$-handling changes associated with metabolic stress impair contractility and are attenuated by AMPK activation. ${ }^{23}$ Thus, metabolic dysfunction and compensation may be important in AF-related contractile changes (Figure 4).

\section{Structural Remodeling}

AF induces atrial structural remodeling, characterized by atrial enlargement and fibrosis. Cardiac fibroblasts are crucial for extracellular matrix deposition and fibrosis. ${ }^{\mathbf{4}}$ Mitochondrial ROS are associated with fibroblast differentiation and cardiac fibrosis, although the mechanisms remain to be clarified. ${ }^{49}$ Several studies reported that oxidative stress stimulates mitogen-activated protein kinase 


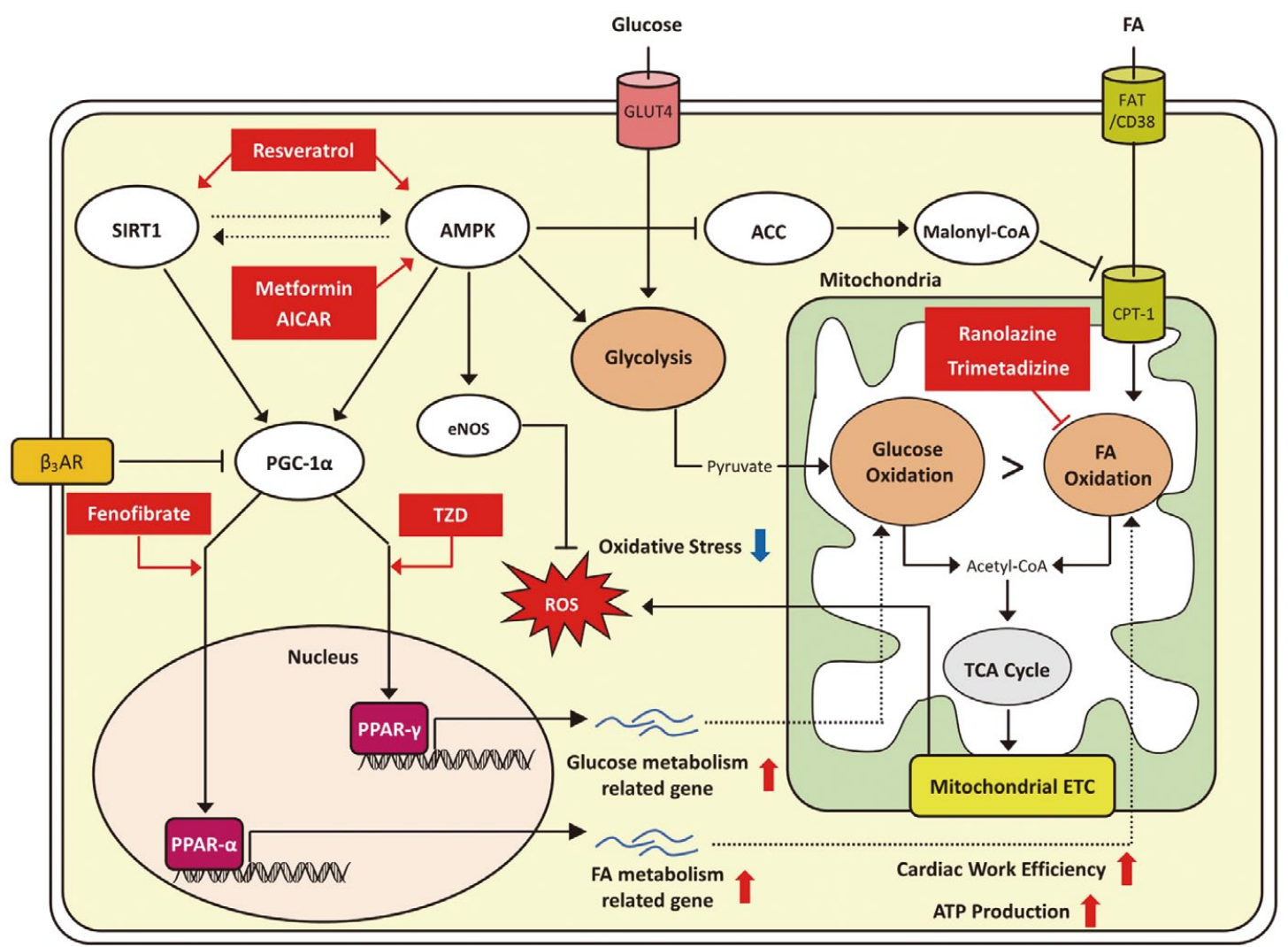

Figure 5. Metabolic modulation and potential therapeutic strategy in AF. ACC, acetyl-CoA carboxylase; $\beta_{3} A R$, $\beta_{3}$-adrenergic receptor; eNOS, endothelial nitric oxide synthase; SIRT1, sirtuin-1; PGC-1a, peroxisome proliferator-activated receptor- $\nu$ coactivator-1 $\alpha$; PPAR- $\alpha / \gamma$, peroxisome proliferator-activated receptor- $\alpha / \gamma$; TGF- $\beta$, transforming growth factor- $\beta$; TZD, thiazolidinedione. Other abbreviations as in Figures 2,4.

and nuclear factor- $\kappa \mathrm{B}(\mathrm{NF}-\kappa \mathrm{B})$ pathways, ${ }^{50,51}$ which interact with $\mathrm{Ca}^{2+}$-mediated profibrotic signaling. The ROS-generator peroxynitrite induces nuclear translocation of $\mathrm{NF}-\kappa \mathrm{B}$ in fibroblasts, leading to fibroblast activation and production of transforming growth factor- $\beta$ (TGF- $\beta$ ), fibronectin, and collagen-I. ${ }^{52}$ ROS also activates profibrotic signaling through Smad 2/3, a downstream target of TGF- $\beta 1 . .^{53}$

Genetic deletion of LKB1, an upstream regulator of AMPK, causes spontaneous AF that progresses into a persistent form in mice, mimicking the human disease process. LKB1 deletion causes dramatic atrial enlargement and fibrosis. ${ }^{54,55}$ AMPK fractional phosphorylation increases in paroxysmal (self-terminating) AF patients but decreases in long-standing persistent AF. ${ }^{23}$ These observations suggest that loss of AMPK-related metabolic adaptation might contribute to atrial structural remodeling and therapeutic resistance to AF (Figure 4).

\section{Metabolic Modulation as a Potential Therapeutic Strategy}

\section{AMPK Activation}

AMPK activation with AICAR improves $\mathrm{Ca}^{2+}$-handling and cell contraction of metabolically stressed atrial cardiomyocytes. ${ }^{23}$ The use of metformin, an AMPK activator, is associated with a decreased risk of AF in patients with type
2 diabetes. ${ }^{56}$ In an vitro study, 4Hz-paced HL-1 atrialderived cardiomyocytes showed increased ROS production and myolysis; treatment with metformin prevented these abnormalities. ${ }^{\mathbf{5 6}}$ These results point to the potential value of AMPK activation in AF therapy.

\section{PPAR-a/PGC-1a Pathway}

Peroxisome proliferator-activated receptor (PPAR)- $\alpha$, a nuclear receptor protein, functions as a transcription factor. PPAR $-\gamma$ coactivator $-1 \alpha$ (PGC- $1 \alpha)$ increases the probability of a gene being transcribed by interacting with PPAR- $\alpha$. Co-activation of PPAR $-\alpha$ and PGC- $1 \alpha$ increases the transcription of FA metabolism-related genes. Sirtuin 1, an anti-aging molecule, and AMPK are upstream regulators of the PPAR- $\alpha /$ PGC- $1 \alpha$ pathway (Figure 5).

Liu et al demonstrated that the protein expression of mCPT-1 and GLUT4 is decreased in atrial tissues from AF patients compared with sinus-rhythm patients, indicating reduced FA oxidation and glucose transport. ${ }^{57}$ The protein expression of sirtuin 1, PGC- $1 \alpha$, and PPAR- $\alpha$ was also decreased in AF. AF rabbits showed a similar decrease in these molecules; treatment with a PPAR- $\alpha$ agonist (fenofibrate) restored the expression of $\mathrm{mCPT}-1$ and GLUT4 and the activation of the PPAR- $\alpha / \operatorname{sirtuin} 1 / \mathrm{PGC}-1 \alpha$ pathway, suppressing AF inducibility. ${ }^{57}$

$\beta_{3}$-adrenergic receptor $\left(\beta_{3}\right.$-AR) activation regulates energy 
metabolism by modulating the PPAR- $\alpha /$ PGC- $1 \alpha$ pathway. In dogs with pacing-induced $\mathrm{AF}, \beta_{3}-\mathrm{AR}$ expression increased and atrial electrical and structural remodeling developed, associated with increased oxidative stress. 58,59 Rapid atrial pacing in rabbits increases the atrial mRNA and protein expression of $\beta_{3}$-ARs, accompanied by reduced atrial APD and increased AF inducibility ${ }^{60}$ These changes are associated with decreased expression of CPT-1, FAT/ CD36, PGC- $1 \alpha$ and PPAR- $\alpha$, resulting in decreased atrial FA concentrations. $\beta_{3}-\mathrm{AR}$ inhibition attenuated electrical remodeling, and improved CTP-1/CD36 expression and PPAR- $\alpha /$ PGC- $1 \alpha$ activation. ${ }^{60}$

Resveratrol, a bioactive polyphenol found in grapes and red wine, has attracted scientific interest because of diverse benefits including antioxidant cardioprotective effects. Resveratrol activates AMPK/sirtuin1 signaling, ${ }^{61}$ modulating cardiac metabolism. A multifunctional smallmolecule resveratrol-derivate compound $(\mathrm{C} 1)$ alters the function of multiple ion channels (IKur, $I_{\mathrm{KACh}}$, and $I_{\mathrm{Na}}$ ) and displays antioxidant properties in human and rat atrial cardiomyocytes. ${ }^{62} \mathrm{C} 1$ treatment also reduced AF sustainability in atrial tachypaced dogs. ${ }^{62}$ In a HF rabbit model, resveratrol activated PI3K/AKT/eNOS signaling and reduced AF susceptibility and triggered activity by preventing atrial electrical, contractile, and fibrotic remodeling. ${ }^{63}$

\section{PPAR- $\gamma$ Pathway}

PPAR- $\gamma$ regulates FA storage and glucose metabolism. Thiazolidinediones (TZDs), such as rosiglitazone and pioglitazone, are potent activators of PPAR- $\gamma$ that have insulin-sensitizing effects via an increase in GLUT4 expression. Pioglitazone prevents atrial electrical and structural remodeling in pacing-induced $\mathrm{HF}$ rabbits with an $\mathrm{AF}$ substrate. ${ }^{64}$ TZD treatment is associated with a decreased risk of new-onset AF in patients with type 2 diabetes. ${ }^{65} \mathrm{In}$ paroxysmal AF patients with type 2 diabetes undergoing catheter ablation, pioglitazone therapy was accompanied by reduced AF recurrence rates. ${ }^{66}$

\section{Inhibition of FA Oxidation}

Cardiac diseases are associated with increased serum catecholamine levels; catecholamines induce lipolysis, increasing the circulating free FA concentration and cellular FA uptake. Increased FA oxidation tends to increase ROS production under hypoxic conditions and uncouples glycolysis from glucose oxidation, which increases the generation of lactate and protons, causing cellular acidosis. Reduction of the FA/glucose metabolism ratio may limit oxygen wastage and improve cardiac efficiency.

Ranolazine was initially developed as an antianginal agent, and has been reported to suppress FA oxidation and to increase glucose oxidation without a concomitant increase in glycolysis or lactate release, improving glycolysis and glucose-oxidation coupling. ${ }^{8}$ Ranolazine has been reported to improve the redox balance and mitochondrial function in AF rats. ${ }^{67}$ Ranolazine also inhibits peak $I_{\mathrm{Na}}$, late $I \mathrm{Na}$, and $I \mathrm{Kr}$ in a dose-dependent manner, and has been shown to suppress ventricular and supraventricular arrhythmia occurrence. ${ }^{68}$ In a phase II trial, ranolazine was safe and well tolerated in patients with persistent AF. ${ }^{69} \mathrm{~A}$ subsequent study showed the combination of ranolazine and dronedarone to be superior to individual drug therapy in suppressing AF occurrence; ${ }^{70}$ however, the relative importance of the metabolic vs. ion-channel actions of the drug is unclear.
Trimetazidine is an antianginal agent that inhibits FA metabolism and improves glucose utilization by blocking 3-ketoacyl-CoA thiolase. Trimetazidine also activates AMPK signaling in the heart, ${ }^{71}$ and might have value in AF treatment.

\section{Conclusions}

There is a close relationship between cellular energy metabolism and AF, which is clearly associated with metabolic stress. Relatively little work has been done to explore the mechanisms and pathophysiological importance of AF-related metabolic stress. A better appreciation of the role of cellular metabolic changes in modulating atrial electrical, structural, and contractile properties in AF might provide important new mechanistic insights and therapeutic opportunities for management of this challenging arrhythmia.

\section{Acknowledgments}

Jennifer Bacchi for excellent secretarial assistance with the manuscript. Supported by the Canadian Institutes of Health Research and the Heart and Stroke Foundation of Canada.

\section{Disclosures}

None.

\section{References}

1. Macle L, Cairns J, Leblanc K, Tsang T, Skanes A, Cox JL, et al. 2016 focused update of the Canadian Cardiovascular Society guidelines for the management of atrial fibrillation. Can J Cardiol 2016; 32: 1170-1185.

2. Heijman J, Algalarrondo V, Voigt N, Melka J, Wehrens XHT, Dobrev D, et al. The value of basic research insights into atrial fibrillation mechanisms as a guide to therapeutic innovation: A critical analysis. Cardiovasc Res 2016; 109: 467-479.

3. Barth AS, Tomaselli GF. Cardiac metabolism and arrhythmias. Circ Arrhythm Electrophysiol 2009; 2: 327-335.

4. Barth AS, Merk S, Arnoldi E, Zwermann L, Kloos P, Gebauer $\mathrm{M}$, et al. Reprogramming of the human atrial transcriptome in permanent atrial fibrillation: Expression of a ventricular-like genomic signature. Circ Res 2005; 96: 1022-1029.

5. Mayr M, Yusuf S, Weir G, Chung YL, Mayr U, Yin X, et al. Combined metabolomic and proteomic analysis of human atrial fibrillation. J Am Coll Cardiol 2008; 51: 585-594.

6. De Souza AI, Cardin S, Wait R, Chung YL, Vijayakumar M, Maguy A, et al. Proteomic and metabolomic analysis of atrial profibrillatory remodelling in congestive heart failure. $\mathrm{J} \mathrm{Mol} \mathrm{Cell}$ Cardiol 2010; 49: 851-863.

7. Tu T, Zhou S, Liu Z, Li X, Liu Q. Quantitative proteomics of changes in energy metabolism-related proteins in atrial tissue from valvular disease patients with permanent atrial fibrillation. Circ J 2014; 78: 993 - 1001 .

8. Lopaschuk GD, Ussher JR, Folmes CD, Jaswal JS, Stanley WC. Myocardial fatty acid metabolism in health and disease. Physiol Rev 2010; 90: 207-258.

9. Jaswal JS, Keung W, Wang W, Ussher JR, Lopaschuk GD. Targeting fatty acid and carbohydrate oxidation: A novel therapeutic intervention in the ischemic and failing heart. Biochim Biophys Acta 2011; 1813: 1333-1350.

10. Fillmore N, Mori J, Lopaschuk GD. Mitochondrial fatty acid oxidation alterations in heart failure, ischaemic heart disease and diabetic cardiomyopathy. Br J Pharmacol 2014; 171: 2080-2090.

11. Cha YM, Dzeja PP, Shen WK, Jahangir A, Hart CY, Terzic A, et al. Failing atrial myocardium: Energetic deficits accompany structural remodeling and electrical instability. Am J Physiol Heart Circ Physiol 2003; 284: H1313-H1320.

12. Ausma J, Wijffels M, Thoné F, Wouters L, Allessie M, Borgers M. Structural changes of atrial myocardium due to sustained atrial fibrillation in the goat. Circulation 1997; 96: 3157-3163.

13. van Bragt KA, Nasrallah HM, Kuiper M, Luiken JJ, Schotten $\mathrm{U}$, Verheule S. Atrial supply-demand balance in healthy adult pigs: Coronary blood flow, oxygen extraction, and lactate 
production during acute atrial fibrillation. Cardiovasc Res 2014; 101: 9-19.

14. Kalifa J, Maixent JM, Chalvidan T, Dalmasso C, Colin D, Cozma D, et al. Energetic metabolism during acute stretchrelated atrial fibrillation. Mol Cell Biochem 2008; 317: 69-75.

15. White CW, Kerber RE, Weiss HR, Marcus ML. The effects of atrial fibrillation on atrial pressure-volume and flow relationship. Circ Res 1982; 51: 205-215.

16. Skalidis EI, Hamilos MI, Karalis IK, Chlouverakis G, Kochiadakis GE, Vardas PE. Isolated atrial microvascular dysfunction in patients with lone recurrent atrial fibrillation. $J$ Am Coll Cardiol 2008; 51: 2053-2057.

17. Barbey O, Pierre S, Duran MJ, Sennoune S, Lévy S, Maixent JM. Specific up-regulation of mitochondrial F0F1-ATPase activity after short episodes of atrial fibrillation in sheep. $J$ Cardiovasc Electrophysiol 2000; 11: 432-438.

18. Ausma J, Coumans WA, Duimel H, Van der Vusse GJ, Allessie MA, Borgers M. Atrial high energy phosphate content and mitochondrial enzyme activity during chronic atrial fibrillation. Cardiovasc Res 2000; 47: 788-796.

19. Lopaschuk GD, Jaswal JS. Energy metabolic phenotype of the cardiomyocyte during development, differentiation, and postnatal maturation. J Cardiovasc Pharmacol 2010; 56: 130-140.

20. Modrego J, Maroto L, Tamargo J, Azcona L, Mateos-Cáceres $\mathrm{P}$, Segura A, et al. Comparative expression of proteins in left and right atrial appendages from patients with mitral valve disease at sinus rhythm and atrial fibrillation. J Cardiovasc Electrophysiol 2010; 21: 859-868.

21. Mihm MJ, Yu F, Carnes CA, Reiser PJ, McCarthy PM, Van Wagoner DR, et al. Impaired myofibrillar energetics and oxidative injury during human atrial fibrillation. Circulation 2001; 104: $174-180$

22. Harada M, Nattel SN, Nattel S. AMP-activated protein kinase: Potential role in cardiac electrophysiology and arrhythmias. Circ Arrhythm Electrophysiol 2012; 5: 860-867.

23. Harada M, Tadevosyan A, Qi X, Xiao J, Liu T, Voigt N, et al. Atrial fibrillation activates AMP-dependent protein kinase and its regulation of cellular calcium handling: Potential role in metabolic adaptation and prevention of progression. $\mathrm{J}$ Am Coll Cardiol 2015; 66: 47-58

24. Lenski M, Schleider G, Kohlhaas M, Adrian L, Adam O, Tian Q, et al. Arrhythmia causes lipid accumulation and reduced glucose uptake. Basic Res Cardiol 2015; 110: 40.

25. Zorov DB, Filburn CR, Klotz LO, Zweier JL, Sollott SJ. Reactive oxygen species (ROS)-induced ROS release: A new phenomenon accompanying induction of the mitochondrial permeability transition in cardiac myocytes. $J$ Exp Med 2000; 192: 1001-1014.

26. Harada M, Van Wagoner DR, Nattel S. Role of inflammation in atrial fibrillation pathophysiology and management. Circ J 2015; 79: $495-502$

27. Takahashi N, Kume O, Wakisaka O, Fukunaga N, Teshima $\mathrm{Y}$, Hara $\mathbf{M}$, et al. Novel strategy to prevent atrial fibrosis and fibrillation. Circ J 2012; 76: 2318-2326.

28. Ausma J, Litjens N, Lenders MH, Duimel H, Mast F, Wouters $\mathrm{L}$, et al. Time course of atrial fibrillation-induced cellular structural remodeling in atria of the goat. $\mathrm{J} \mathrm{Mol} \mathrm{Cell} \mathrm{Cardiol}$ 2001; 33: 2083-2094.

29. Carnes CA, Chung MK, Nakayama T, Nakayama H, Baliga RS, Piao S, et al. Ascorbate attenuates atrial pacing-induced peroxynitrite formation and electrical remodeling and decreases the incidence of postoperative atrial fibrillation. Circ Res 2001; 89: e32-e38.

30. Schild L, Bukowska A, Gardemann A, Polczyk P, Keilhoff G, Täger M, et al. Rapid pacing of embryoid bodies impairs mitochondrial ATP synthesis by a calcium-dependent mechanism: A model of in vitro differentiated cardiomyocytes to study molecular effects of tachycardia. Biochim Biophys Acta 2006; 1762: $608-615$.

31. Lamirault G, Gaborit N, Le Meur N, Chevalier C, Lande G, Demolombe $\mathrm{S}$, et al. Gene expression profile associated with chronic atrial fibrillation and underlying valvular heart disease in man. J Mol Cell Cardiol 2006; 40: 173-184.

32. Seppet E, Eimre M, Peet N, Paju K, Orlova E, Ress M, et al. Compartmentation of energy metabolism in atrial myocardium of patients undergoing cardiac surgery. Mol Cell Biochem 2005; 270: $49-61$.

33. Emelyanova L, Ashary Z, Cosic M, Negmadjanov U, Ross G, Rizvi F, et al. Selective downregulation of mitochondrial electron transport chain activity and increased oxidative stress in human atrial fibrillation. Am J Physiol Heart Circ Physiol 2016; 311:
$\mathrm{H} 54-\mathrm{H} 63$.

34. Montaigne D, Marechal X, Lefebvre P, Modine T, Fayad G, Dehondt $\mathrm{H}$, et al. Mitochondrial dysfunction as an arrhythmogenic substrate: A translational proof-of-concept study in patients with metabolic syndrome in whom post-operative atrial fibrillation develops. J Am Coll Cardiol 2013; 62: 1466-1473.

35. Tsuboi M, Hisatome I, Morisaki T, Tanaka M, Tomikura Y, Takeda S, et al. Mitochondrial DNA deletion associated with the reduction of adenine nucleotides in human atrium and atrial fibrillation. Eur J Clin Invest 2001; 31: 489-496.

36. Park HW, Ahn Y, Jeong MH, Cho JG, Park JC, Kang JC, et al. Chronic atrial fibrillation associated with somatic mitochondrial DNA mutations in human atrial tissue. J Clin Pathol 2007; 60: 948-950.

37. Lai LP, Tsai CC, Su MJ, Lin JL, Chen YS, Tseng YZ, et al. Atrial fibrillation is associated with accumulation of agingrelated common type mitochondrial DNA deletion mutation in human atrial tissue. Chest 2003; 123: 539-544.

38. Weiss JN, Lamp ST. Glycolysis preferentially inhibits ATPsensitive $\mathrm{K}+$ channels in isolated guinea pig cardiac myocytes. Science 1987; 238: 67-69.

39. Wijffels MC, Kirchhof CJ, Dorland R, Power J, Allessie MA Electrical remodeling due to atrial fibrillation in chronically instrumented conscious goats: Roles of neurohumoral changes, ischemia, atrial stretch, and high rate of electrical activation. Circulation 1997; 96: 3710-3720.

40. Brundel BJ, Van Gelder IC, Henning RH, Tuinenburg AE, Wietses M, Grandjean JG, et al. Alterations in potassium channel gene expression in atria of patients with persistent and paroxysmal atrial fibrillation: Differential regulation of protein and mRNA levels for K+ channels. J Am Coll Cardiol 2001; 37: 926-932.

41. Balana B, Dobrev D, Wettwer E, Christ T, Knaut M, Ravens U. Decreased ATP-sensitive $\mathrm{K}(+)$ current density during chronic human atrial fibrillation. J Mol Cell Cardiol 2003; 35: 1399-1405.

42. Sánchez C, Corrias A, Bueno-Orovio A, Davies M, Swinton J, Jacobson I, et al. The $\mathrm{Na}+/ \mathrm{K}+$ pump is an important modulator of refractoriness and rotor dynamics in human atrial tissue. $\mathrm{Am}$ J Physiol Heart Circ Physiol 2012; 302: H1146-H1159.

43. Workman AJ, Kane KA, Rankin AC. Characterisation of the $\mathrm{Na}, \mathrm{K}$ pump current in atrial cells from patients with and without chronic atrial fibrillation. Cardiovasc Res 2003; 59: 593-602.

44. Tran CT, Schmidt TA, Christensen JB, Kjeldsen K. Atrial $\mathrm{Na}, \mathrm{K}-\mathrm{ATPase}$ increase and potassium dysregulation accentuate the risk of postoperative atrial fibrillation. Cardiology 2009; 114: $1-7$.

45. Stary V, Puppala D, Scherrer-Crosbie M, Dillmann WH, Armoundas AA. SERCA2a upregulation ameliorates cellular alternans induced by metabolic inhibition. J Appl Physiol 2016; 120: $865-875$.

46. Erickson JR, Joiner ML, Guan X, Kutschke W, Yang J, Oddis $\mathrm{CV}$, et al. A dynamic pathway for calcium-independent activation of CaMKII by methionine oxidation. Cell 2008; 133: 462-474.

47. Fisher M, McAllister M. Cardiological aspects of stroke prevention. Circ J 2015; 79: 271-277.

48. Yue L, Xie J, Nattel S. Molecular determinants of cardiac fibroblast electrical function and therapeutic implications for atrial fibrillation. Cardiovasc Res 2011; 89: 744-753.

49. Friedrichs K, Baldus $\mathrm{S}$, Klinke A. Fibrosis in atrial fibrillation: Role of reactive species and MPO. Front Physiol 2012; 3: 214.

50. Clerk A, Fuller SJ, Michael A, Sugden PH. Stimulation of "stress regulated" mitogen-activated protein kinases (stress-activated protein kinases/c-Jun N-terminal kinases and p38-mitogenactivated protein kinases) in perfused rat hearts by oxidative and other stresses. J Biol Chem 1998; 273: 7228-7234.

51. Frantz S, Kelly RA, Bourcier T. Role of TLR-2 in the activation of nuclear factor kappa B by oxidative stress in cardiac myocytes. $J$ Biol Chem 2001; 276: 5197-5203.

52. Ichikawa T, Sugiura H, Koarai A, Yanagisawa S, Kanda M, Hayata A, et al. Peroxynitrite augments fibroblast-mediated tissue remodeling via myofibroblast differentiation. Am J Physiol Lung Cell Mol Physiol 2008; 295: L800-L808.

53. Cucoranu I, Clempus R, Dikalova A, Phelan PJ, Ariyan S, Dikalov S, et al. NAD(P)H oxidase 4 mediates transforming growth factor-beta1-induced differentiation of cardiac fibroblasts into myofibroblasts. Circ Res 2005; 97: 900-907.

54. Ikeda Y, Sato K, Pimentel DR, Sam F, Shaw RJ, Dyck JR, et al. Cardiac-specific deletion of LKB1 leads to hypertrophy and dysfunction. J Biol Chem 2009; 284: 35839-35849.

55. Ozcan C, Battaglia E, Young R, Suzuki G. LKB1 knockout mouse develops spontaneous atrial fibrillation and provides 
mechanistic insights into human disease process. $J$ Am Heart Assoc 2015; 4: e001733.

56. Chang SH, Wu LS, Chiou MJ, Liu JR, Yu KH, Kuo CF, et al. Association of metformin with lower atrial fibrillation risk among patients with type 2 diabetes mellitus: A population-based dynamic cohort and in vitro studies. Cardiovasc Diabetol 2014; 13: 123 .

57. Liu GZ, Hou TT, Yuan Y, Hang PZ, Zhao JJ, Sun L, et al. Fenofibrate inhibits atrial metabolic remodelling in atrial fibrillation through PPAR- $\alpha$ /sirtuin 1/PGC- $1 \alpha$ pathway. $\mathrm{Br} J$ Pharmacol 2016; 173: 1095-1109.

58. Yu J, Li W, Li Y, Zhao J, Wang L, Dong D, et al. Activation of $\beta(3)$-adrenoceptor promotes rapid pacing-induced atrial electrical remodeling in rabbits. Cell Physiol Biochem 2011; 28: 87-96.

59. Sheng L, Shen Q, Huang K, Liu G, Zhao J, Xu W, et al. Upregulation of $\beta 3$-adrenergic receptors contributes to atrial structural remodeling in rapid pacing induced atrial fibrillation canines. Cell Physiol Biochem 2012; 30: 372-381.

60. Liu Y, Geng J, Liu Y, Li Y, Shen J, Xiao X, et al. $\beta 3$-adrenoceptor mediates metabolic protein remodeling in a rabbit model of tachypacing-induced atrial fibrillation. Cell Physiol Biochem 2013; 32: $1631-1642$.

61. Kanamori H, Takemura G, Goto K, Tsujimoto A, Ogino A, Takeyama $\mathrm{T}$, et al. Resveratrol reverses remodeling in hearts with large, old myocardial infarctions through enhanced autophagy-activating AMP kinase pathway. Am J Pathol 2013; 182: $701-713$.

62. Baczko I, Liknes D, Yang W, Hamming KC, Searle G, Jaeger K, et al. Characterization of a novel multifunctional resveratrol derivative for the treatment of atrial fibrillation. Br J Pharmacol 2014; 171: 92-106.

63. Chong E, Chang SL, Hsiao YW, Singhal R, Liu SH, Leha T, et al. Resveratrol, a red wine antioxidant, reduces atrial fibrillation susceptibility in the failing heart by PI3K/AKT/eNOS signaling pathway activation. Heart Rhythm 2015; 12: 1046-1056.

64. Shimano M, Tsuji Y, Inden Y, Kitamura K, Uchikawa T,
Harata S, et al. Pioglitazone, a peroxisome proliferator-activated receptor-gamma activator, attenuates atrial fibrosis and atrial fibrillation promotion in rabbits with congestive heart failure. Heart Rhythm 2008; 5: 451-459.

65. Chao TF, Leu HB, Huang CC, Chen JW, Chan WL, Lin SJ, et al. Thiazolidinediones can prevent new onset atrial fibrillation in patients with non-insulin dependent diabetes. Int J Cardiol 2012; 156: $199-202$.

66. Gu J, Liu X, Wang X, Shi H, Tan H, Zhou L, et al. Beneficial effect of pioglitazone on the outcome of catheter ablation in patients with paroxysmal atrial fibrillation and type 2 diabetes mellitus. Europace 2011; 13: 1256-1261.

67. Zou D, Geng N, Chen Y, Ren L, Liu X, Wan J, et al. Ranolazine improves oxidative stress and mitochondrial function in the atrium of acetylcholine- $\mathrm{CaCl} 2$ induced atrial fibrillation rats. Life Sci 2016; 156: 7-14.

68. Scirica BM, Morrow DA, Hod H, Murphy SA, Belardinelli L, Hedgepeth CM, et al. Effect of ranolazine, an antianginal agent with novel electrophysiological properties, on the incidence of arrhythmias in patients with non ST-segment elevation acute coronary syndrome: Results from MERLIN-TIMI 36 randomized controlled trial. Circulation 2007; 116: 1647-1652.

69. De Ferrari GM, Maier LS, Mont L, Schwartz PJ, Simonis G, Leschke M, et al. Ranolazine in the treatment of atrial fibrillation: Results of the dose-ranging RAFFAELLO study. Heart Rhythm 2015; 12: $872-878$

70. Reiffel JA, Camm AJ, Belardinelli L, Zeng D, KarwatowskaProkopczuk E, Olmsted A, et al. Combined ranolazine and dronedarone in the management of paroxysmal atrial fibrillation: Mechanistic and therapeutic synergism. Circ Arrhythm Electrophysiol 2015; 8: 1048-1056.

71. Liu Z, Chen JM, Huang H, Kuznicki M, Zheng S, Sun W, et al. The protective effect of trimetazidine on myocardial ischemia/ reperfusion injury through activating AMPK and ERK signaling pathway. Metabolism 2016; 65: 122-130. 